\title{
Cabinet médical sous l'eau? Que faire?
}

\section{L'assurance du cabinet médical pour tous les cas}

Lundi matin après un reposant week-end. Une nouvelle semaine vous attend, votre agenda est rempli de rendez-vous de patients. Quelle surprise lorsque vous franchissez la porte de votre cabinet. Toutes vos pièces sont sous l'eau. Comment voulez-vous continuer à travailler?

\section{FMH Insurance Services vous aide avec son assurance pour le cabinet}

Souhaitons que vous ayez contracté une assurance FMH Insurance Services pour votre cabinet. Un coup de téléphone suffit et les premières mesures sont prises. Votre assurance couvre les dommages, tels que ceux causés par l'incendie, les événements naturels (p.ex. inondations, avalanches, éboulement de rochers, etc.), les dégâts d'eau, le vol avec effraction et le détroussement sont inclus. De plus vous pouvez assurer la perte d'exploitation qui résulte de ce sinistre.

\section{Solutions d'assurances selon votre besoin spécifique}

Selon votre spécialité vos besoins d'assurances sont différents. FMH Insurance Services peut les adapter et vous offrir la flexibilité nécessaire. Différentes assurances complémentaires, p.ex. I'assurance des appareils médicaux, ainsi que le coffre en cas d'urgence peuvent être conclues.

\section{Primes attractives pour les membres FMH}

L'assurance du cabinet de la FMH Insurance Services vous offre une relation prix-prestations optimum. Une comparaison du marché montre que la concurrence est jusqu'à $40 \%$ plus chère.

\section{Profitez maintenant et commandez une offre personnelle}

Vos besoins sont au centre de nos préoccupations. Notre nom vous garantit le conseil professionnel de nos conseillers qui possèdent une longue expérience dans le domaine des assurances, des produits bancaires et financiers. Grâce à notre indépendance, nous pouvons vous offrir les meilleurs produits adaptés à vos besoins.

\section{Talon réponse}

Prénom / Nom

Adresse

NPA / Lieu

Date de naissance

Téléphone privé/cabinet

Atteignable le plus facilement (heure)

Adresse e-mail

Prière de $m^{\prime}$ 'envoyer une offre $d^{\prime}$ 'assurance de cabinet FMH Insurance Services (merci de joindre la copie de votre police actuelle)

Je désire un conseil personnalisé Prière de me contacter

Je suis intéressé aux produits suivants:

$\begin{array}{ll}\text { Placement financier } & \text { Caisse de pension LPP } \\ \text { Pilier } 3 a & \bigcirc \text { Protection juridique } \\ \text { Planification financière } & \text { O Responsabilité civile profession. } \\ & \end{array}$

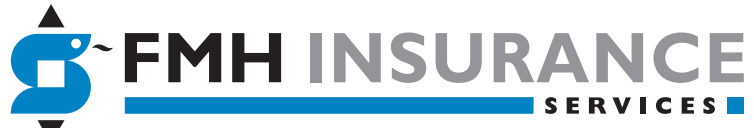

Roth Gygax \& Partner AG $\mathbf{\square}$ Service de coordination Moosstrasse 2 घ 3073 Gümligen

Téléphone 0319595000 • Fax 0319595010 mail@fmhinsurance.ch 匹 www.fmhinsurance.ch 